\title{
Patrones de consumo de mariguana, abastecimiento y estado de ánimo durante el confinamiento por COVID- I 9
}

\author{
Paulina Beverido Sustaetaㅁ', Betzaida Salas-Garcíaㅁ', Xóchitl De San Jorge-Cárdenas(D', María del Carmen \\ Gogeascoechea-Trejo(D), Patricia Pavón-León(D', María Leticia Sobeida Blázquez-Morales(D', Alma de los Ángeles

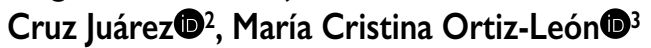 \\ I Instituto de Ciencias de la Salud de la Universidad Veracruzana, México \\ 2 Dirección General del Área de Ciencias de la Salud de la Universidad Veracruzana, México \\ 3 Instituto de Salud Pública de la Universidad Veracruzana, México
}

\section{RESUMEN}

Introducción: el surgimiento de la pandemia por el virus SARS-CoV-2 ha generado cambios en los estilos de vida de la humanidad. El distanciamiento y cuarentena, estrategias adoptadas para prevenir el contagio y evitar el colapso de los sistemas de salud, han afectado a las personas de distintas maneras de acuerdo con su propia vulnerabilidad, entre ellos a los consumidores de mariguana. Objetivo: conocer si los consumidores habituales de mariguana que permanecieron en aislamiento físico y social debido al COVID-19, presentaron modificaciones o cambios en su consumo, en el estado de ánimo y en la forma en que se abastecieron. Método: estudio transversal, no probabilístico y analítico, que utilizó la técnica bola de nieve dirigido a consumidores habituales de mariguana en México, mayores de edad, que cuentan con internet y/o WhatsApp. Resultados: participaron 50 hombres y 25 mujeres, la mayor parte del estado de Veracruz; la única variable relacionada con el estado de ánimo que resultó estadísticamente significativa fue el alivio que proporciona el consumo de mariguana y nivel de consumo durante la cuarentena; también se registraron cambios en el uso, en la forma de abastecimiento y en la relación establecida con las personas con que conviven. Conclusiones: este trabajo aborda una problemática emergente de la que aún se conoce poco, especialmente en lo que se refiere a su interrelación con la salud mental y con los trastornos por el uso de sustancias psicoactivas, por lo que contribuye a construir una base para la comprensión del impacto de la cuarentena y para la atención de los consumidores de mariguana.

\begin{abstract}
ABCTRACT
Introduction: the emergence of the pandemic caused by the SARS-Cov-2 virus has generated lifestyles changes for humanity. Strategies to prevent exponential contagion and the collapse of health systems such as social-distancing and quarantine have affected people in different ways according to their vulnerability, including marijuana consumers. Objective: to learn if the regular consumers of marijuana who remained in physical and social isolation due to COVID-19 altered or modified their consumption, in their disposition and in their method of supply. Method: a cross-sectional, non-probabilistic and analytical study that used the snowball technique, aimed at regular marijuana consumers in Mexico, of legal age, who have access to the Internet and/or WhatsApp. Results: 50 men and 25 women participated, most from the state of Veracruz; the only mood related variable that was statistically significant was the relief provided by marijuana use and level of marijuana use during quarantine, as well there were changes in consumption, in the method of supply and in the relationship established with the people with whom they live. Conclusions: this work addresses an emerging problem of which little is still known, especially regarding its correlation with mental health and disorders resulting from the use of psychoactive substances. Thus, contributing a building basis for understanding the impact of quarantine and for the attention of marijuana consumers.
\end{abstract}

Keywords: marijuana use; social isolation; quarantine; COVID-19.

Palabras clave: uso de mariguana; aislamiento social; cuarentena; COVID-19.

\footnotetext{
Autor de correspondencia:

María Cristina Ortiz León. Av. Doctor Luis Castelazzo Ayala S/N, Industrial Animas, C.P. 91190, Xalapa Enríquez, Ver., México Teléfono: 2288421700 ext. 13303. Correo electrónico: cortiz@uv.mx

Recibido: 23 de junio de 2020

Aceptado: 8 de septiembre de 2020

doi: 10.28931/riiad.2020.2.04
} 


\section{INTRODUCCIÓN}

La situación de emergencia debido a la pandemia ocasionada por el virus SARS-CoV-2 ha provocado un drástico cambio en el estilo de vida de la mayoría de los habitantes del mundo; el aislamiento físico y social -distanciamiento y cuarentena- se consolidan como las mejores medidas adoptadas en el mundo para la prevención del contagio y para aplanar la curva epidémica, con el fin de evitar la saturación de los sistemas de salud (Medeiros de Figueiredo et al., 2020). La implementación de estas medidas registra diversos matices que van de lo voluntario a lo obligatorio, lo cual afecta de distintas maneras a los miembros de cada comunidad.

La Organización Mundial de la Salud (OMS) ha estado llamando la atención sobre las consideraciones especiales que merece la salud mental durante y después de ésta o cualquier emergencia (Álvarez-Monjarás \& Bucay-Harari, 2018) y ha advertido que esta pandemia puede estar afectando doblemente a la población consumidora de sustancias psicoactivas, debido a la presencia de los síndromes de abstinencia, moderados o severos, aunado a un estado de ánimo alterado ante las dificultades para abastecerse de drogas y consumirlas.

De acuerdo con Volkow (2006), el consumo de drogas altera diversas áreas del cerebro, particularmente aquellas vinculadas al análisis, expresión y control emocional, planificación del comportamiento, control ejecutivo, toma de decisiones y condicionamiento del miedo; esto es importante ya que aunque no existen estudios sobre los efectos del aislamiento social que está viviendo la población durante esta pandemia; empiezan a publicarse reportes que indican que puede provocar síntomas de depresión y estrés y otros estados de ánimo desagradables, como la soledad y el aburrimiento, considerados como factores de riesgo para que inicie o aumente el consumo de sustancias psicoactivas o se registre reincidencia después de haberlas dejado. El riesgo se incrementa si se trata de personas que viven el confinamiento solas o sin una red de apoyo social (Borja, 2020).

Entre estos reportes destaca el emitido por el subdirector general de drogodependencias de la Conselleria de Salud de la Generalitat de Catalunya (Europa Press, 2020), quien subrayó la necesidad de mantener activada -durante la pandemia- la red de atención para las personas con adicciones, dado que se trata de un colectivo de alta vulnerabilidad: el funcionario estima que hay menos droga en circulación, menos oferta y menos consumo en Cataluña, lo que puede traducirse en un incremento de la ansiedad y el estrés así como en el descontrol de las emociones, ello impacta en el sistema inmunológico y aumenta el riesgo de infección.
Este estudio, se enfoca en el consumo de mariguana durante la pandemia del SARS-CoV-2, debido a que junto con el tabaco y el alcohol- es la droga de mayor consumo en el mundo y el panorama puede tornarse aún más grave, ya las personas adictas tienen mayor riesgo de contraer otras enfermedades y porque tienen un acceso limitado al cuidado de la salud debido a la estigmatización y exclusión de la que generalmente son objeto, situación que puede exacerbarse ante la saturación de la capacidad de clínicas y hospitales como resultados del COVID-19. En este contexto, el National Institute on Drug Abuse (NIDA, 2020), alertó que "...dado que el coronavirus que causa COVID-19 ataca los pulmones, podría representar un peligro particularmente grave para quienes vapean o fuman tabaco o mariguana".

A pesar de lo anterior, un reporte del Bank of America (Elceo, 2020) informó que la venta de mariguana y productos cannábicos en Estados Unidos se incrementó en 90\% durante el confinamiento por COVID-19 en el mes de marzo, y que los estados que cuentan con expendios autorizados de estos productos fueron considerados como "servicios esenciales", por lo que siguieron operando normalmente; quizá por lo mismo, en los hospitales de Estados Unidos se ha observado un incremento notable en la incidencia de brotes psicóticos desde que empezó la pandemia, pasando de uno a cuatro casos mensuales, lo que resulta preocupante ya que como advirtió el Dr. Jyoti K. Randhawa, director médico del Hospital del Comportamiento Silver Oaks, en New Lenox (Illinois), "más de 30 por ciento de las personas que tienen psicosis inducida por el cannabis desarrollarán algún tipo de psicosis más adelante en la vida" (Baran, 2020). Asimismo, neumólogos de la Asociación Americana del Pulmón llaman la atención sobre el consumo temprano y crónico de mariguana, ya que el primero provoca una inflamación seria de las vías respiratorias y el segundo, bronquitis, lo que dificulta mucho el diagnóstico de COVID-19 (LaMotte, 2020).

En Colombia se aplicó una encuesta a consumidores de todas las drogas y en abril se publicó un informe que identificó que la mariguana ha sido la sustancia de mayor consumo durante la cuarentena (25\%), y que los usuarios la relacionan con mayor productividad en el teletrabajo (17\%); $83 \%$ de los 1,249 participantes tienen entre $18 \mathrm{y}$ 29 años de edad, lo que coincide con los resultados de estudios epidemiológicos publicados en Latinoamérica, respecto a que se trata del grupo poblacional con mayor consumo (Corporación Acción Técnica Social, 2020).

Es de esperarse que en esta situación se agudice el consumo de sustancias con presencia de cuadros de ansiedad (Kalawski, 2020) y que el aislamiento provoque modificaciones o cambios en el consumo y en el estado de ánimo de las personas. Por ello, desde una 
perspectiva de reducción del daño, y para prevenir contagios por COVID-19 entre los consumidores de mariguana, el comisionado nacional contra las adicciones de México, Gady Zabicky Sirot, recomendó evitar compartir el cigarrillo o el dispositivo que se use para fumar la droga (Suárez, 2020). Ante la ausencia de alternativas para el cuidado de usuarios vulnerables por parte de la Secretaría de Salud y de la Comisión Nacional contra las Adicciones (CONADIC), los consumidores están publicando recomendaciones a través de diversos blogs, en los que hacen un llamamiento a "...si no dejamos de fumar, podemos hacerlo en menos cantidad o usar alternativas, como ingerirla en alimentos; además de evitar compartir la parafernalia y el consumo colectivo... Igual de responsable será continuar cultivando para mantener el abasto..." (La dosis, 2020).

Derivado de lo anterior, el objetivo del estudio fue conocer si los consumidores habituales de mariguana presentaron modificaciones o cambios en su consumo, en el estado de ánimo y en la forma en que se abastecieron derivados del aislamiento físico y social debido al COVID-19.

\section{MÉTODO}

El estudio fue de carácter transversal, no probabilístico y analítico, dirigido a consumidores habituales de mariguana que viven en territorio mexicano, mayores de 18 años y que cuentan con internet y/o WhatsApp, ya que derivado de las condiciones del confinamiento se decidió realizar la recolección de la información de manera online, lo que también favorecía el anonimato y elegir el momento y el lugar de contestar el instrumento. La liga para acceder al cuestionario, publicado en la plataforma Typeform, se difundió desde el estado de Veracruz a través de correo electrónico y WhatsApp entre consumidores de mariguana con los que se tenía un contacto previo.

Debido a su aplicación en línea y ante la ausencia de un marco muestral que permitiera obtener una muestra representativa de consumidores de mariguana, se eligió el muestreo bola de nieve, el cual es el más recomendado cuando se trata del estudio de asuntos particularmente delicados o privados que implican cierta estigmatización (Rao, 2017), como el caso del consumo de mariguana o cuando la población es de difícil acceso (Siddiqui, 2016), por lo que se consideró que el recibir la invitación de algún conocido, sintiera confianza para participar en el estudio. La invitación especificaba que el cuestionario era breve, anónimo y con fines académicos y pedía que lo compartieran con otros consumidores mayores de edad que conocieran, que podían estar dentro o fuera de la entidad; dada la falta de restricción en cuanto a quiénes se deberían enviar, el muestreo bola de nieve fue no discriminatorio exponencial. El cuestionario estuvo disponible por 31 días -del 17 de abril al 17 de mayo de 2020- correspondientes al primer mes de la Fase 3 de la epidemia en México, caracterizada por un rápido ascenso del contagio, la acumulación de casos positivos y hospitalizaciones, que obligaron a intensificar la cuarentena en el marco de la Jornada Nacional de Sana Distancia.

El cuestionario, diseñado ad hoc, cuenta con 10 ítems que indagan aspectos sociodemográficos (edad, sexo, entidad federativa); variaciones en el consumo de mariguana; dificultades para el abasto; cambios en el estado de ánimo y en las relaciones interpersonales con quienes conviven los participantes. Al inicio de este, se desplegaba un mensaje en donde se especificaba que sólo en caso de ser mayor de 18 años podía participar. Este cuestionario fue validado por un grupo de expertos, dado que no se consideró medición ni cuantificación de un constructo, no se aplicó ningún procedimiento estadístico para evaluar su validez (Frías-Navarro, 2019).

Para el análisis de los datos se utilizó estadística descriptiva con cálculo de frecuencias, porcentajes y medidas de tendencia central. Se consideró como variable de interés la cantidad de mariguana consumida durante el confinamiento, con tres categorías de respuesta: incremento, disminución o igual. La estadística inferencial se usó para comprobar la asociación entre el nivel de cantidad de mariguana consumida respecto de las variables cualitativas, por lo que se aplicó la prueba Ji-cuadrada $\left(X^{2}\right)$; en el caso de las variables numéricas se aplicó la prueba de Kolmogorov-Smirnorv para comprobar la normalidad de los datos, debido a que ninguna de estas variables cumplía con este supuesto se utilizó además la prueba de Kruskal-Wallis.

Para comprobar si el número de cigarrillos de mariguana -antes y durante el confinamiento, tanto entre semana como en fin de semana- había variado, se aplicó la prueba no paramétrica de rangos con signo de Wilcoxon $(W$, ya que tampoco esta variable cumplió con el supuesto de normalidad. El valor que se consideró significativo fue $p<0.05$. Los resultados fueron analizados con el software estadístico IBM SPSS, versión 24.

Durante el proceso de investigación, se consideraron los aspectos éticos establecidos en el Reglamento de la Ley General de Salud en materia de investigación para la salud, incluyendo el anonimato y la autonomía de los participantes, quienes recibieron información oportuna sobre el objetivo de la encuesta y sus fines académicos. Al finalizar la encuesta, cada participante recibió información sobre las opciones de atención, consejería y tratamiento en línea. 


\section{RESULTADOS}

Participaron 75 consumidores habituales de mariguana, $67 \%$ hombres y $33 \%$ mujeres, con edades de 20 a 66 años; más del 50\% tenía menos de 27 años; el grupo de edad en el que se concentró el mayor número de cuestionarios fue de 20 a 25 años (41\%) y la edad más frecuente fue de 23 años. Alrededor de $40 \%$ de los participantes se encontraba en el estado de Veracruz y el $60 \%$ restante en otras entidades federativas: Querétaro, Ciudad de México, Oaxaca, Estado de México, Quintana Roo, Puebla, Chiapas, Baja California y Guerrero; más de la mitad se encontraban confinados con sus familias (53\%) y la mayoría $(74.6 \%)$ declaró estar en cuarentena casi total durante el período evaluado, lo que significa salir únicamente por despensa y alimentos.
El análisis de resultados muestra que las características sociodemográficas y el tipo de confinamiento que tuvieron una asociación estadísticamente significativa con el nivel de mariguana consumida fue: entidad en donde se llevó a cabo el confinamiento y personas con quienes vivía. En la Tabla 1, se muestra que el consumo de mariguana durante el confinamiento se incrementó en aquellos que viven fuera de la entidad veracruzana $(52.3 \%)$ respecto a los que viven en el estado de Veracruz (22.6\%), lo que fue estadísticamente significativo $\left(X^{2}=11.998, p=.002\right)$. Respecto a las personas con quienes vivía el usuario de mariguana se encontró que los que viven con pareja o con amigos, reportaron un incremento en el consumo respecto a los que viven con la familia, que reportaron consumir menos $\left(X^{2}=19.521\right.$, $p=.003)$.

Tabla 1

Características sociodemográficas y tipo de confinamiento según el nivel de consumo de mariguana durante el distanciamiento físico y social por COVID-19, 2020

\begin{tabular}{|c|c|c|c|c|c|}
\hline Variable & Total & Fumó más & Fumó menos & Igual & Estadístico/p \\
\hline Sexo * & & & & & $.000 / 1.0$ \\
\hline Hombres & $50(100)$ & $20(40)$ & $16(32)$ & $14(28)$ & \\
\hline Mujeres & $25(100)$ & $10(40)$ & $8(32)$ & $7(28)$ & \\
\hline Edad ** & $27(32-23)$ & $28(24.8-30.8)$ & $24.5(23-29.3)$ & $29(34-25)$ & $5.736 / .057$ \\
\hline Grupos de edad * & & & & & $10.083 / .121$ \\
\hline 20 a 25 años & $31(100)$ & $10(22.6)$ & $14(45.2)$ & $7(22.6)$ & \\
\hline 26 a 30 años & $24(100)$ & $13(54.2)$ & $6(25.0)$ & $5(20.8)$ & \\
\hline 31 a 35 años & $14(100)$ & $5(35.7)$ & $4(28.6)$ & $5(35.7)$ & \\
\hline Más de 35 años & $6(100)$ & $2(33.3)$ & - & $4(66.7)$ & \\
\hline \multicolumn{5}{|c|}{ Lugar en donde se encuentra en confinamiento * } & $11.998 / .002$ \\
\hline Veracruz & $31(100)$ & $7(22.6)$ & $9(29.0)$ & 15 (48.4) & \\
\hline Otro lugar & $44(100)$ & 23 (52.3) & $15(34.1)$ & $6(13.6)$ & \\
\hline Con quién vive * & & & & & $19.521 / .003$ \\
\hline Con familia & $40(100)$ & $10(25.0)$ & $20(50.0)$ & $10(25.0)$ & \\
\hline Solo & $14(100)$ & $6(42.9)$ & $1(7.1)$ & $7(50.0)$ & \\
\hline Con amigos & $12(100)$ & 7 (58.3) & $3(25.0)$ & $2(16.7)$ & \\
\hline Con pareja & $9(100)$ & $7(77.8)$ & - & $2(22.2)$ & \\
\hline
\end{tabular}


Tabla 1

Características sociodemográficas y tipo de confinamiento según el nivel de consumo de mariguana durante el distanciamiento físico y social por COVID-19, 2020 (continuación)

\section{Tipo de confinamiento *}

$4.703 / 7.582$

$\begin{array}{lcccc}\text { Total } & 9(100) & 2(22.2) & 4(44.4) & 3(33.3) \\ \text { Casi total } & 56(100) & 22(39.3) & 19(33.9) & 15(26.8) \\ \text { Parcial } & 9(100) & 5(55.6) & 1(11.1) & 3(33.3) \\ \text { Vida normal } & 1(100) & 1(100) & - & -\end{array}$

Notas: En negrita se encuentran los resultados estadísticamente significativos

* Los datos se representan como n (\%). Se realizó la prueba de Ji-Cuadrada.

** Los datos se representan como Mediana (Tercer cuartil-Primer cuartil). Se realizó la prueba de Kruskal-Wallis.

Fuente: elaboración propia a partir de los datos obtenidos.

Con relación al número de cigarros de mariguana consumidos entre semana -antes y durante el confinamiento- en la Tabla 2 se aprecia que hubo un aumento estadísticamente significativo entre quienes manifestaron fumar más, ya que de tres cigarrillos pasaron a cinco ( $W=-2.551, p=.011)$. En cambio, hubo una reducción en el consumo entre quienes declararon fumar menos, al pasar de 4.5 a un cigarrillo, lo que resultó estadísticamente significativo ( $W=-3.600, p<.001)$. En cuanto al consumo en fin de semana, de manera general se aprecia una reducción estadísticamente significativa $(W=-2.682, p=$ .007) de tres a dos en el grupo que informó estar consumiendo más y también en el grupo que declaró consumir menos, ya que pasó de 2.5 a $0.5(W=-3.827, p<.001)$.

Tabla 2

Consumo de mariguana antes y durante el distanciamiento físico y social, 2020

\begin{tabular}{|c|c|c|c|c|}
\hline Variable & $\begin{array}{c}\text { Total } \\
N=75\end{array}$ & $\begin{array}{l}\text { Fumó más } \\
\qquad n=30\end{array}$ & $\begin{array}{l}\text { Fumó menos } \\
\qquad n=24\end{array}$ & $\begin{array}{l}\text { Igual } \\
n=21\end{array}$ \\
\hline \multicolumn{5}{|l|}{ Mediana de cigarrillos consumidos* } \\
\hline Entre semana antes del distanciamiento social & $3(10.0-1.4)$ & $3(7-2)$ & $4.5(10-1.1)$ & $2(10-1)$ \\
\hline Entre semana durante el distanciamiento social & $3(6.0-1.0)$ & $5(7.3-3)$ & $1(4-0)$ & $2(10-1)$ \\
\hline Wilcoxon & -0.667 & -2.551 & -3.600 & 0.000 \\
\hline Valor-p & .504 & .011 & $<.001$ & 1.00 \\
\hline Fines de semana antes del distanciamiento social & $3(4.0-1.0)$ & $3(5.3-1.8)$ & $2.5(4.75-1)$ & $3(4-1)$ \\
\hline Fines de semana durante el distanciamiento social & $2(0.5-4.0)$ & $4(5.3-2)$ & $0.5(1.5-0)$ & $2(4.5-1)$ \\
\hline Wilcoxon & -2.682 & -1.764 & -3.827 & -0.946 \\
\hline Valor $\mathrm{p}$ & .007 & .078 & $<.001$ & .344 \\
\hline
\end{tabular}

Notas: * Los datos se representan como Mediana (Tercer cuartil-Primer cuartil). Se realizó la prueba de los rangos con signo de Wilcoxon ( $W$. En negrita se encuentran los resultados estadísticamente significativos.

Fuente: elaboración propia a partir de los datos recabados. 
En la Tabla 3, se abordan las variables sobre adquisición de mariguana, antes y durante la cuarentena, que tienen una asociación estadísticamente significativa con el nivel de mariguana consumida: cambio en las formas de adquirir la mariguana en confinamiento, forma de adquirirla durante el distanciamiento físico y social y razón del cambio en la adquisición o en el consumo.

De los encuestados, $60 \%$ cambió su manera de conseguir mariguana debido al confinamiento, sin importar el nivel de consumo reportado. En la misma tabla se observa que aquellos que reportaron un consumo igual, antes y durante el confinamiento, mostraron un porcentaje mayor de cambio (76\%), en comparación con quienes reporta- ron consumir menos (38\%), lo que resultó estadísticamente significativo $\left(X^{2}=7.912 p=.019\right)$. La principal manera de conseguir la droga antes del confinamiento fue la compra a narcomenudistas o dealers (75\%); durante el confinamiento $35 \%$ mantuvo esta forma de abastecimiento y $25 \%$ no contestó esta pregunta. El grupo de quienes fumaban menos reportó haber agotado pronto sus reservas (17\%), con resultados estadísticamente significativos ( $X^{2}$ $=34.97, p=.001$ ). Las principales razones del cambio en la adquisición y/o en el consumo fueron: no poder fumar en donde se encontraban y la dificultad para conseguirla, con $17 \%$ respectivamente, aunque $45 \%$ no contestó esta pregunta $\left(X^{2}=19.77, p=.011\right)$.

Tabla 3

Forma de adquisición de la mariguana antes y durante el confinamiento según el nivel de consumo durante el distanciamiento físico y social, 2020

\begin{tabular}{|c|c|c|c|c|c|}
\hline Variable & $\begin{array}{c}\text { Total } \\
N=75\end{array}$ & $\begin{array}{l}\text { Fumó más } \\
\qquad n=30\end{array}$ & $\begin{array}{l}\text { Fumó menos } \\
\qquad n=24\end{array}$ & $\begin{array}{l}\text { Igual } \\
n=21\end{array}$ & Estadístico/p \\
\hline \multicolumn{5}{|c|}{ Cambió la forma de adquirir la mariguana en confinamiento * } & 7.912/.019 \\
\hline Sí & $45(60)$ & $20(67)$ & $9(38)$ & $16(76)$ & \\
\hline No & $30(40)$ & $10(33)$ & $15(62)$ & $5(24)$ & \\
\hline \multicolumn{5}{|c|}{ Forma de adquirirla antes del distanciamiento físico y social * } & $5.261 / .262$ \\
\hline Narcomenudistas o dealers & $56(75)$ & $25(83)$ & $17(71)$ & $14(67)$ & \\
\hline Compartían amigos & $15(20)$ & $3(10)$ & $7(29)$ & $5(24)$ & \\
\hline Cultivaba en casa & $4(5)$ & $2(7)$ & $0(0)$ & $2(9)$ & \\
\hline \multicolumn{5}{|c|}{ Forma de adquirirla después del distanciamiento físico y social * } & $34.97 / .001$ \\
\hline Mismo proveedor & $26(35)$ & $10(33)$ & $11(46)$ & $5(24)$ & \\
\hline A domicilio & $4(5)$ & $1(3)$ & $1(4)$ & $2(10)$ & \\
\hline Agotar reserva & $5(7)$ & $1(3)$ & $4(17)$ & $0(0)$ & \\
\hline $\begin{array}{l}\text { Comparten amigos o } \\
\text { familiares }\end{array}$ & $8(11)$ & $5(17)$ & $3(13)$ & $0(0)$ & \\
\hline Compro en otro lado & $9(12)$ & $6(20)$ & $3(13)$ & $0(0)$ & \\
\hline No consigo & $3(4)$ & $0(0)$ & $2(8)$ & $1(5)$ & \\
\hline Otra razón & $1(1)$ & $0(0)$ & $0(0)$ & $1(5)$ & \\
\hline No contestó & $19(25)$ & $7(23)$ & $0(0)$ & $12(57)$ & \\
\hline \multicolumn{5}{|c|}{ Razón del cambio en la adquisición o en el consumo* } & $19.77 / .011$ \\
\hline $\begin{array}{l}\text { No puedo fumar en donde } \\
\text { me encuentro }\end{array}$ & $13(17)$ & $0(0)$ & $9(38)$ & $4(19)$ & \\
\hline Es más difícil conseguirla & $13(17)$ & $4(13)$ & $4(17)$ & $5(24)$ & \\
\hline $\begin{array}{l}\text { Prefiero no salir a conseguirla } \\
\text { por precaución }\end{array}$ & $10(13)$ & $6(20)$ & $4(17)$ & $0(0)$ & \\
\hline Otra razón & $5(7)$ & $3(10)$ & $1(4)$ & $1(5)$ & \\
\hline No contestaron & $34(45)$ & $17(57)$ & $6(25)$ & $11(52)$ & \\
\hline
\end{tabular}

Notas: * Los datos se representan como n (\%). Se realizó la prueba de Ji-Cuadrada $\left(X^{2}\right)$.

En negrita se encuentran los resultados estadísticamente significativos.

Fuente: elaboración propia a partir de los datos recabados. 
En la Tabla 4, se presentan algunos elementos del estado de ánimo durante la cuarentena, como resultado del aislamiento físico y social. La respuesta que concentró mayor porcentaje (36\%) fue haberse sentido ansioso durante la cuarentena y $71 \%$ refirió que fumar mariguana aliviaba este malestar. $67 \%$ de quienes expresaron fumar más manifestaron que el alivio que sentían al fumar mariguana ayudaba a que desapareciera su ansiedad, esta fue la única variable relacionada con el estado de ánimo que resultó estadísticamente significativa $\left(X^{2}=\right.$ 16.01, $p=.042)$. La gran mayoría (64\%), manifestó que el consumo de mariguana en esta etapa no había alterado sus relaciones con las personas con las que compartía la cuarentena.

Tabla 4

Estado de ánimo durante el confinamiento por COVID-19 según nivel de consumo de mariguana, 2020

\begin{tabular}{|c|c|c|c|c|c|}
\hline Variable & $\begin{array}{c}\text { Total } \\
N=75\end{array}$ & $\begin{array}{l}\text { Fumó más } \\
\qquad n=30\end{array}$ & $\begin{array}{l}\text { Fumó menos } \\
\qquad n=24\end{array}$ & $\begin{array}{l}\text { Igual } \\
n=21\end{array}$ & Estadístico/p \\
\hline \multicolumn{5}{|c|}{ Estado de ánimo por el distanciamiento físico y social * } & $8.520 / .578$ \\
\hline Ansioso & $27(36)$ & $13(43)$ & $5(25)$ & $8(38)$ & \\
\hline Aburrido & $14(19)$ & $4(13)$ & $7(29)$ & $4(14)$ & \\
\hline Miedo & $6(8)$ & $2(7)$ & $2(8)$ & $2(9.5)$ & \\
\hline Deprimido & $5(7)$ & $2(7)$ & $1(4)$ & $2(9.5)$ & \\
\hline Igual que antes & $16(21)$ & $4(13)$ & $7(29)$ & $5(24)$ & \\
\hline Otro estado de ánimo & $7(9)$ & $5(17)$ & $1(4)$ & $1(5)$ & \\
\hline \multicolumn{5}{|c|}{ Sensaciones al consumir mariguana durante el distanciamiento físico y social * } & $7.709 / .103$ \\
\hline Lo alivia & $53(71)$ & $26(87)$ & $15(63)$ & $12(57)$ & \\
\hline Lo hace sentir igual & $17(23)$ & $4(13)$ & $7(29)$ & $6(29)$ & \\
\hline No lo alivia & $5(6)$ & $0(0)$ & $2(8)$ & $3(14)$ & \\
\hline \multicolumn{5}{|c|}{ Alivio que proporciona el consumo de mariguana * } & $16.01 / .042$ \\
\hline Desaparece la ansiedad & $36(48)$ & $20(67)$ & $8(33)$ & $8(38)$ & \\
\hline Disminuye el miedo & $5(7)$ & $2(7)$ & $1(4)$ & $2(10)$ & \\
\hline Aumenta la relajación & $9(12)$ & $3(10)$ & $1(4)$ & $5(24)$ & \\
\hline Otras & $3(4)$ & $1(3)$ & $2(8)$ & $0(0)$ & \\
\hline No contestó & $22(29)$ & $4(13)$ & $12(50)$ & $6(29)$ & \\
\hline \multicolumn{5}{|c|}{$\begin{array}{l}\text { Impacto del consumo de mariguana en las relaciones con otras personas } \\
\text { durante el distanciamiento físico y social }{ }^{*}\end{array}$} & $7.478 / .113$ \\
\hline Las ayuda & $24(32)$ & $13(43)$ & $8(33)$ & $3(14)$ & \\
\hline Las empeora & $3(4)$ & $1(3)$ & $2(8)$ & $0(0)$ & \\
\hline Son iguales & $48(64)$ & $16(64)$ & $14(58)$ & $18(86)$ & \\
\hline
\end{tabular}

Notas: * Los datos se representan como $n(\%)$. Se realizó la prueba de Ji-Cuadrada $\left(\mathrm{X}^{2}\right)$.

En negrita se encuentran los resultados estadísticamente significativos.

Fuente: elaboración propia a partir de los datos recabados.

\section{DISCUSIÓN Y CONCLUSIONES}

Lidiar con una situación nueva, urgente, de duración indefinida, que pone en riesgo la vida e incrementa la incertidumbre en el futuro, como la derivada de la pandemia por COVID-19, ha obligado a la mayor parte de los países del mundo a adoptar medidas sanitarias como el aislamiento físico y social, que afectan a la población en general y a los distintos grupos vulnerables.

La vulnerabilidad ha sido definida como la condición interna de un sujeto o grupo expuesto a una amenaza/ evento traumático, que resulta de un proceso dinámico de interacción de diversos factores, entre los que destaca la disposición intrínseca para ser dañado. La 
Organización Panamericana de la Salud (2006) advirtió que una gran cantidad de enfermos y muertes, la falta de equipamiento en clínicas y hospitales y las enormes pérdidas económicas en el contexto de una epidemia o pandemia generan un riesgo psicosocial elevado.

Este estudio se ocupó de conocer la manera en que las condiciones de aislamiento sugeridas por el gobierno federal y los gobiernos estatales en México han afectado a los consumidores de drogas, específicamente de mariguana, por ser considerados un colectivo de alta vulnerabilidad, ya que además de la adicción, enfrentan un mayor riesgo debido a la forma predominante de consumo: fumada o vapeada (NIDA, 2020). El análisis de casos realizado por Wu y McGoogan (2020), con información proveniente de los Centros para el Control y la Prevención de Enfermedades de China, indica que las muertes por COVID-19 se dieron sobre todo en hombres con Enfermedad Pulmonar Obstructiva Crónica (EPOC) provocada por la exposición e ingesta del humo derivado del consumo de drogas, sin distinguir si era de tabaco, mariguana o alguna otra substancia. Los autores consideran que es necesario continuar investigando para clarificar los distintos factores relacionados tanto con la susceptibilidad al COVID-19 como con su evolución clínica.

Este análisis tiene algunas limitantes, tales como la brevedad de la medición; el escaso número de participantes y la concentración mayoritaria de éstos en dos de las entidades federativas del país: Veracruz y Querétaro. Sin ánimo de justificación, es oportuno recordar que el cuestionario no se publicó en ninguna plataforma o red social, sólo pasó de un conocido a otro; estas limitaciones son adjudicables a la técnica de bola de nieve, que permitió alcanzar únicamente a un grupo de consumidores que se conocían entre sí, lo que muestra la interconexión entre usuarios de mariguana.

Sin embargo, el estudio obtuvo resultados interesantes, uno de ellos es que desde el inicio de la pandemia y en la cuarentena no ha disminuido la oferta ni el consumo de mariguana en México, a diferencia de la contracción reportada en Cataluña por la Conselleria de Salud de la Generalitat de Catalunya (Europa Press, 2020), aunque sin alcanzar lo reportado por Baran (2020) en Estados Unidos respecto al incremento del consumo que ha aumentado el número de brotes psicóticos por cannabis atendidos.

En lo que corresponde al estado de ánimo y su relación con el consumo, los resultados coinciden con lo establecido por Kalawski (2020) cuando señala que el consumo agudo se acompaña de la presencia de ansiedad; lo mismo sucedió en el caso de México, en donde la mayor parte de los consumidores que participaron atribuyeron este estado de ánimo a la cuarentena y reportaron un alivio al fumar mariguana. También, los datos obteni- dos en este trabajo concuerdan con lo publicado por Borja, (2020) cuando refiere que el aislamiento provoca estados de ánimo alterados, sobre todo en las personas que viven solas. Esta situación permite concluir que el aislamiento es un factor de riesgo para el consumo de drogas, aunque en el caso de México no se tengan resultados estadísticamente significativos, además el momento en que se realizó el estudio fue al inicio de la cuarentena, por lo que los datos reflejados en el estado de ánimo de los usuarios de mariguana debido al confinamiento resultan poco concluyentes.

El uso de mariguana se incrementó entre aquellos que viven solos o con amigos, lo que coincide con lo reportado en Borja, (2020). En relación con la disponibilidad y accesibilidad a esta sustancia, los hallazgos son distintos a los reportados por el Bank of America (Elceo, 2020) en Estados Unidos, ya que allá los puntos de distribución se consideraron como servicios esenciales, mientras que en México sigue siendo provista por dealers debido a que todavía es una sustancia ilícita para la mayor parte de los consumidores.

Otro resultado importante del trabajo es que permitió descubrir que por lo menos $5 \%$ de los consumidores cultivan la mariguana que van a consumir, lo que revela estrategias de autocuidado en el ámbito de la seguridad pública, coincidentes con las recomendadas en el blog La dosis (2020).

Los demás indicadores no fueron comparados con otros debido a que se aborda una problemática no sólo reciente sino con características que únicamente habían sido experimentadas hace poco más de un siglo, cuando la humanidad enfrentó la crisis sanitaria derivada de la pandemia de la llamada gripa española, que llevó a Soper (1919) a escribir: "Inundaciones, hambrunas, terremotos y erupciones volcánicas han dejado sin excepción huellas demasiado terribles como para entenderlas, pero aún así nunca había habido una catástrofe a la vez tan repentina, tan devastadora y tan universal".

Esta experiencia dio sustento a una de las recomendaciones más importantes de la OMS en el marco de su Plan de Preparación para la Pandemia de Gripe (2006), en el que sugería que todos los países establecieran un Comité Ejecutivo Nacional para la preparación y ejecución del Plan de Actuación y preveía que el tratamiento para esta infección estaría enfocado en tratar los síntomas que la enfermedad produce. Una de las recomendaciones, dirigida a los sistemas de salud del mundo, incluidas en este plan de actuación, se refería a evitar que las personas afectadas por el virus en turno consumieran alcohol y tabaco; en otras palabras, no contemplaba el consumo de otras drogas como la mariguana, a pesar de que ya en el año 2006 era la tercera droga de mayor consumo, in- 
dependientemente de su estatus legal en algunos países o prohibida en la mayoría.

Como ha señalado NIDA (2020), a pesar de que hay numerosos grupos e investigadores trabajando en el tema, aún se sabe poco sobre el COVID-19 y menos sobre la manera en que se interrelaciona con los trastornos mentales, particularmente con los derivados del consumo de drogas. Sin embargo, resulta adecuado utilizar los conocimientos y experiencias resultantes del estudio de la relación entre el consumo de drogas, sobre todo de las que son fumadas o vapeadas, en la salud de las personas. En este marco, resulta claro que trabajos como este sientan las bases para profundizar en el estudio y comprensión de una problemática que parece será de larga duración y a la que habrá que dar respuestas oportunas y pertinentes, basadas en evidencias, para evitar agravar la salud de los mexicanos con trastornos en la salud mental.

\section{FUENTES DE FINANCIAMIENTO}

No se obtuvieron fondos externos para la realización del presente estudio.

\section{DECLARACIONES DE LOS AUTORES}

No existen conflictos de interés relacionados con el manuscrito.

\section{REFERENCIAS}

Álvarez-Monjarás, M. \& Bucay-Harari, L. (2018). Modelo de tamizaje, atención y monitoreo de la salud mental para adultos en casos de desastre. Salud Pública de México, 60(supl. 1), S23-S30. doi: 10.21149/9340

Baran, D. (5 de junio de 2020). Los casos de psicosis inducida por el cannabis aumentan durante la pandemia de COVID-19. News Tribune. https://www.newstrib.com/2020/06/01/cases-of-cannabis-induced-psychosis-increase-during-covid-19-pandemic/axa9jtv/

Borja, M. (13 de abril de 2020). Cómo afrontar una adicción, legal o ilegal, al estar confinado. Lasdrogas.info. https://www.lasdrogas. info/noticias/como-afrontar-una-adiccion-legal-o-ilegal-al-estarconfinado-el-riesgo-de-recaer-aumenta-en-los-que-viven-solos/

Corporación Acción Técnica Social. (2020). Échele Cabeza. Informe sobre el consumo de drogas en cuarentena. http://www.echelecabeza.com/informe-sobre-el-consumo-de-drogas-en-cuarentena/

Elceo. (6 de abril de 2020). Consumo de cannabis aumenta en EU debido a confinamiento por coronavirus. https://elceo.com/negocios/consumo-de-cannabis-aumenta-en-eu-por-confinamiento-debido-a-coronavirus/

Europa Press. (13 de abril de 2020). Los adictos a las drogas consumen más alcohol y fármacos durante el confinamiento del coronavirus. Infosalus. https://www.infosalus.com/salud-investigacion/ noticia-adictos-drogas-consumen-mas-alcohol-farmacos-confinamiento-coronavirus-20200413105147.html

Frías-Navarro, D. (2019). Apuntes de consistencia interna de las puntuaciones de un instrumento de medida. Universidad de Valencia, 1-13. Recuperado de: https://www.uv.es/friasnav/AlfaCronbach.pdf

Kalawski, J. (5 de mayo de 2020). COVID-19: Recommendations for drug treatment programs. https://www.oas.org/ext/en/main/covid-19/Virtual-Forums/ArtMID/3425/ArticleID/2753/COVID-19-Recommendations-for-drug-treatment-programs

La dosis. (2 de abril de 2020). ¿Fumar o no fumar marihuana en tiempos de COVID-19? https://ladosis.org/articulos/fumar-o-no-fumarmarihuana-en-tiempos-de-covid-19/

LaMotte, S. (10 de abril de 2020). Fumar marihuana y coronavirus: incluso el uso ocasional aumenta el riesgo de complicaciones de COVID-19. CNN. https://cnnespanol.cnn.com/2020/04/10/fumar-marihuana-y-coronavirus-incluso-el-uso-ocasional-aumenta-el-riesgo-de-complicaciones-de-covid-19/

Medeiros de Figueiredo, A., Daponte, A., Marculino de Figueiredo, D.C.M., Gil-García, E., \& Kalache, A. (2020). Letalidad del COVID-19: ausencia de patrón epidemiológico, Gaceta Sanitaria. doi: 10.1016/j.gaceta.2020.04.001

National Institute on Drug Abuse. (2020). COVID-19: Las posibles implicaciones para las personas con trastornos por consumo de drogas. https://www.drugabuse.gov/es/about-nida/noras-blog/covid-19-las-posibles-implicaciones-para-las-personas-con-trastornos-por-consumo-de-drogas

Organización Panamericana de la Salud. (2006). Protección de la Salud Mental en Condiciones de Epidemia. THS/MH/06/. Documento elaborado por la Unidad de Salud Mental, Abuso de Sustancias y Rehabilitación (THS/MH) Tecnología y Prestación de Servicios de Salud (THS).https://www.paho.org/hq/dmdocuments/2009/Pandemia\%20de\%20influenza\%20y\%20Salud\%20 mental\%20Esp.pdf

Rao, A., Stahlman, S., Hargreaves, J., Weir, S., Edwards, J., Rice, B., Kochelani, D., Mavimbela, M. \& Baral, S. (2017). Sampling Key Populations for HIV Surveillance: Results From Eight Cross-Sectional Studies Using Respondent-Driven Sampling and Venue-Based Snowball Sampling. JMIR Public Health and Surveillance, 3(4), e72. doi: 10.2196/publichealth.8116

Siddiqui, N. A., Rabidas, V. N., Sinha, S. K., Verma, R. B., Pandey, K., Singh, V. P., Ranjan, A., Topno, R. K., Lal, C. S., Kumar, V., Sahoo, G. C., Sridhar, S., Pandey, A. \& Das, P. (2016). Snowball Vs. House-to-House Technique for Measuring Annual Incidence of Kala-azar in the Higher Endemic Blocks of Bihar, India: A Comparison. PLoS Neglected Tropical Diseases, 10(9), 1-15. https://doi.org/10.1371/journal.pntd.0004970

Soper, G. (1919). The lessons of the pandemic. Science, 49(1274), 501-506. Doi: 10.1126/science.49.1274.501

Suárez, G. (14 abril de 2020). Piden no dar "el toque y rol" entre los consumidores de marihuana para evitar Covid-19. El Heraldo de México. https://heraldodemexico.com.mx/pais/consumidores-de-marihuana-piden-evitar-reuniones-contagio-de-covid-conadic/ 
Volkow, N. (2006). Conferencia inaugural Congreso CPDD/2006. Washington, NIDA, citada en A. Molina. (2013). Personas, emociones y adicciones: Más allá del cerebro. Revista Adicción y Ciencia, 3(1).
Wu, Z. \& McGoogan, JM. (2020). Characteristics of and Important Lessons From the Coronavirus Disease 2019 (COVID-19) Outbreak in China: Summary of a Report of 72314 Cases From the Chinese Center for Disease Control and Prevention. JAMA, 323(13), 1239-1242. Doi: 10.1001/jama.2020.2648 